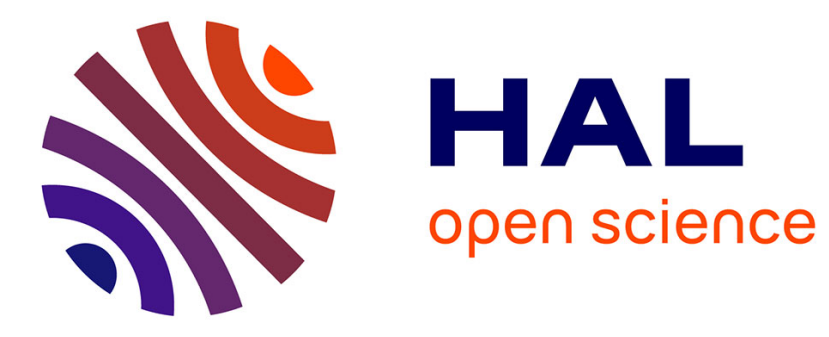

\title{
Multi-Criteria Decision Making Method with Belief Preference Relations
}

\author{
Amel Ennaceur, Zied Elouedi, Eric Lefevre
}

\section{To cite this version:}

Amel Ennaceur, Zied Elouedi, Eric Lefevre. Multi-Criteria Decision Making Method with Belief Preference Relations. International Journal of Uncertainty, Fuzziness and Knowledge-Based Systems, 2014, 22 (04), pp.573-590. 10.1142/S0218488514500287 . hal-03354151

\section{HAL Id: hal-03354151 \\ https://hal.science/hal-03354151}

Submitted on 29 Sep 2021

HAL is a multi-disciplinary open access archive for the deposit and dissemination of scientific research documents, whether they are published or not. The documents may come from teaching and research institutions in France or abroad, or from public or private research centers.
L'archive ouverte pluridisciplinaire HAL, est destinée au dépôt et à la diffusion de documents scientifiques de niveau recherche, publiés ou non, émanant des établissements d'enseignement et de recherche français ou étrangers, des laboratoires publics ou privés. 


\title{
Multi-Criteria Decision Making Method with Belief Preference Relations
}

\author{
Amel Ennaceur \\ University of Tunis, Institut Supérieur de Gestion de Tunis, \\ LARODEC, Tunis, Tunisia \\ amel_naceur@yahoo.fr \\ Zied Elouedi \\ University of Tunis, Institut Supérieur de Gestion de Tunis, \\ LARODEC, Tunis, Tunisia \\ zied.elouedi@gmx.fr \\ Eric Lefevre \\ Univ. Lille Nord of France, UArtois EA 3926 LGI2A, France \\ eric.lefevre@univ-artois.fr \\ Received (received date) \\ Revised (revised date)
}

\begin{abstract}
In modeling Multi-Criteria Decision Making (MCDM) problem, we usually assume that the decision maker is able to elicitate his preferences with precision and without difficulty. However, in many situations, the expert is unable to provide his assessment with certainty or he is unwilling to quantify his preferences. To deal with such situations, a new MCDM model under uncertainty is introduced. In fact, we focus here on the problem of modeling expert opinions despite the presence of incompleteness and uncertainty in their preference assessments. Besides, our proposed solution suggests to model these preferences qualitatively rather than exact numbers. Therefore, we propose to incorporate belief preference relations into a MCDM method. The expert assessments are then formulated as a belief function problem since this theory is considered as a useful tool to model expert judgments.
\end{abstract}

Keywords: Multi-criteria decision making, belief function theory, Belief pair-wise comparison, Uncertain preferences

\section{Introduction}

The Analytic Hierarchy Process (AHP) method ${ }^{78}$ is amongst one of the most well known multi-criteria decision making (MCDM) methods and has been successfully applied to many practical problems. ${ }^{6} 29$ Though its popularity and efficiency, this method is often criticized for its use of an unbalanced scale of estimations and its inability to adequately handle the uncertainty and imprecision associated with the mapping of the decision maker's perception to a crisp number. ${ }^{1} 2$ Besides, in MCDM process, an expert may be uncertain about his level of preference due to incomplete information or knowledge, inherent complexity and uncertainty within the decision environment. Thus, in some cases, the decision maker cannot estimate his assessment with a numerical value. 
To overcome these difficulties, several MCDM methods are combined within uncertain theories. In particular, the AHP is extended to a more realistic elicitation procedure. One of these extensions is the Fuzzy AHP appeared in ${ }^{9}$, which utilized triangular fuzzy numbers to model the pair-wise comparisons. Since then, several fuzzy AHP developments have been proposed. ${ }^{10}$ Besides, probabilistic AHP methods are introduced in ${ }^{11}$, handling pairwise comparisons matrices based on probability theory, where each element of which is the prior probability. There are also other ways to solve AHP problems, such as referenced AHP. ${ }^{12}$

In particular in the belief function framework, Beynon et al. have proposed a method called the DS/AHP method ${ }^{13}$ comparing not only singleton alternatives but also groups of alternatives. Besides, several works has been proposed by Utkin. ${ }^{18}{ }^{19} \mathrm{He}$ has proposed a method for ranking of alternatives in the frameworks of AHP approach and belief function theory. The main feature of this approach is that it allows the expert to deal with comparisons of arbitrary subsets of alternatives and criteria. ${ }^{30}$ Additionally, Smarandache et al. ${ }^{22}$ have developed the DSmT/AHP which is based on the Dezert-Smarandache theory. ${ }^{15}$ This method aimed at performing a similar purpose as DS/AHP that is to compare groups of alternatives. Besides, Ennaceur et al. ${ }^{5}$ have proposed the belief AHP approach that compares groups of criteria to subsets of alternatives. Then, they model the causality relationship between these groups of alternatives and criteria. ${ }^{28}$

With regard to these proposed methods, we can frequently find limits. Firstly, in some cases, the decision maker might be unwilling to provide all comparisons necessary to construct full comparison matrices. In addition, these approaches deal only with numerical values to translate the expert preferences into quantitative information.

To solve the problems presented above, and to facilitate the pair-wise comparison process, a new MCDM method under uncertainty is proposed that eliminates some of the drawbacks of the existing prioritization methods. The main objective of this paper is then to develop a new approach to tackle uncertainty and imprecision within the pair-wise comparison process, in particular, when the decision maker's judgments are represented as a qualitative assessment. A natural way to cope with uncertain judgments is to express the comparison ratios as a belief function, which incorporates the imperfection of the human thinking. Indeed, preferential assessments are used in order to express the decision maker's subjective assessments instead of using numerical values. With our method, the expert uses only preference relations. He does not require to complete all the comparison matrices, he can then derive priorities from incomplete set of judgments. Therefore, a new procedure is employed to derive crisp priorities from qualitative judgments, corresponding to each level.

In what follows, we first present some definitions needed for belief function context. Next, we describe some existing methods for constructing belief function from preference relations. Section 4 details our new MCDM method based on preferential assessment, and gives an example to show its application. Finally, section 5 concludes the paper. 


\section{Belief function theory}

The belief function theory is appropriate to handle uncertainty in MCDM problems especially within approaches based on expert assessments. So, in this section, we briefly review the main concepts underlying the belief function theory as interpreted by the Transferable Belief Model (TBM). The latter is a useful model to represent quantified belief functions. Details can be found in. ${ }^{3} 17$

\subsection{Basic concepts}

Let $\Theta$ be the frame of discernment representing a finite set of elementary hypotheses related to a problem domain. We denote by $2^{\Theta}$ the set of all the subsets of $\Theta$. $^{3}$

The impact of a piece of evidence on the different subsets of the frame of discernment $\Theta$ is represented by the so-called basic belief assignment (bba), called initially by Shafer, basic probability assignment: ${ }^{3}$

$$
\sum_{A \subseteq \Theta} m(A)=1 .
$$

The value $m(A)$, named a basic belief mass (bbm), represents the portion of belief committed exactly to the event $A$. The events having positive bbm's are called focal elements. Let $\mathcal{F}(m) \subseteq 2^{\Theta}$ be the set of focal elements of the bba $m$.

Associated with $m$ is the belief function is defined for $A \subseteq \Theta$ and $A \neq \emptyset$ as:

$$
\operatorname{bel}(A)=\sum_{\emptyset \neq B \subseteq A} m(B) \text { and } \operatorname{bel}(\emptyset)=0 .
$$

The degree of belief $\operatorname{bel}(A)$ given to a subset $A$ of the frame $\Theta$ is defined as the sum of all the basic belief masses given to subsets that support $A$ without supporting its negation.

The plausibility function $p l$ expresses the maximum amount of specific support that could be given to a proposition $A$ in $\Theta$. It measures the degree of belief committed to the propositions compatible with $A . p l(A)$ is then obtained by summing the bbm's given to the subsets $B$ such that $B \cap A \neq \emptyset:{ }^{3}$

$$
p l(A)=\sum_{B \cap A \neq \emptyset} m(B), \quad \forall A \subseteq \Theta .
$$

\subsection{Combination}

Handling information induced from different experts (information sources) requires an evidence gathering process in order to get the fused information. In the TBM, the basic belief assignments induced from distinct pieces of evidence can be combined using the conjunctive rule: ${ }^{16}$

$$
\left(m_{1} @ m_{2}\right)(A)=\sum_{B, C \subseteq \Theta, B \cap C=A} m_{1}(B) m_{2}(C), \quad \forall A \subseteq \Theta .
$$

$m_{1} @ m_{2}$ is the bba representing the combined impact of two pieces of evidence. 


\subsection{Decision making}

The TBM considers that holding beliefs and making decision are distinct processes. Hence, it proposes a two level model:

- The credal level where beliefs are entertained and represented by belief functions.

- The pignistic level where beliefs are used to make decisions and represented by probability functions called the pignistic probabilities, denoted BetP: ${ }^{4}$

$$
\operatorname{BetP}(A)=\sum_{B \subseteq \Theta} \frac{|A \cap B|}{|B|} \frac{m(B)}{(1-m(\emptyset))}, \forall A \in \Theta .
$$

\subsection{Uncertainty measures}

In the case of the belief function framework, the bba is defined on an extension of the powerset: $2^{\Theta}$ and not only on $\Theta$. In the powerset, each element is not equivalent in terms of precision. Indeed, $\theta_{i} \subset \Theta(i \in\{1,2\})$ is more precise than $\theta_{1} \cup \theta_{2} \subseteq \Theta$.

In order to try to quantify this imprecision, different uncertainty measures have been defined, such as ${ }^{26} 27$ :

$$
H(m)=\sum_{A \in \mathcal{F}(m)} m(A) \log _{2}\left(\frac{|A|}{m(A)}\right) .
$$

The measure $H$ is aimed at assessing the total uncertainty arising in a body of evidence due to both randomness (ignorance and inconsistency) and nonspecificity associated with a bba.

The measure of uncertainty $H$ based on a noncomposite approach to total uncertainty, leads to a function that can be factored into the sum of Dubois and Prade's nonspecificity and an entropy-like measure associated with random sets that was previously studied by Nguyen. Under complete ignorance, $H$ reduces to Hartley's information. ${ }^{27}$ Besides, when the bba concentrates only on singletons, this measure is equivalent to Shannon's probabilistic entropy. Besides, $H$ has a unique maximum, in sharp contradistinction to the composite measures $G_{1}, G_{2}$ (called global uncertainty measures ${ }^{31}$ ) and $T$ (called total uncertainty ${ }^{32}$ ). In addition, the measure $H$ attains its global maximum when the bba distributes both randomness and nonspecificity uniformly over the largest possible set of focal elements.

\subsection{Operations on the product space}

In the previous subsections, we have presented the basic concepts of the belief function theory. These mechanisms are based on the assumption that bbas are defined on the same frame of discernment. However, this constraint limits the practical applications. Let us consider in what follows, a first frame $\Theta$ and a second frame $\Omega$.

\subsubsection{Cylindrical extension and projection}

Cylindrical extension : allows to extend a set defined in low-dimensional domain into a higher-dimensional domain. Consider a subset $A \subseteq \Theta$, the cylindrical extension of $A$ to 
$\Theta \times \Omega$ is denoted $A^{\uparrow \Theta \times \Omega}$. It is obtained as:

$$
A^{\uparrow \Theta \times \Omega}=A \times \Omega .
$$

Example: Let us consider $\Theta=\left\{\theta_{1}, \theta_{2}\right\}$. We want to define $\theta_{1}$ into the two-dimensional space $\Theta \times \Omega$ where $\Omega=\left\{\omega_{1}, \omega_{2}\right\}$. Its cylindrical extension is computed as:

$$
\theta_{1}^{\uparrow \Theta \times \Omega}=\left\{\left(\theta_{1}, \omega_{1}\right),\left(\theta_{1}, \omega_{2}\right)\right\} .
$$

Projection: is the opposite operation of cylindrical extension. It allows to reduce a set defined in a multi-dimensional domain to a set defined in a lower-dimensional domain. Let $C$ be a subset of $\Theta \times \Omega$. Projecting $C$ on $\Omega$, denoted $C^{\downarrow \Omega}$, means dropping extra coordinates. It is obtained by:

$$
C^{\downarrow \Omega}=\left\{\omega, \omega \in \Omega, C \cap \omega^{\uparrow \Omega \times \Theta} \neq \emptyset\right\} .
$$

Example: Let us consider $\left\{\left(\theta_{1}, \omega_{1}\right),\left(\theta_{2}, \omega_{1}\right)\right\}$ defined on $\Theta \times \Omega$. The projection of this set into $\Omega$ is equal to: $\left\{\left(\theta_{1}, \omega_{1}\right),\left(\theta_{2}, \omega_{1}\right)\right\}^{\downarrow \Omega}=\omega_{1}$.

\subsubsection{Vacuous Extension}

This operation is useful, when the referential is changed by adding new variables. Thus, a marginal mass function $m^{\Theta}$ defined on $\Theta$ will be expressed in the frame $\Theta \times \Omega$ as follows: 21

$$
m^{\Theta \uparrow \Theta \times \Omega}(C)=\left\{\begin{array}{l}
m^{\Theta}(A) \text { if } C=A \times \Omega, A \subseteq \Theta, \\
0 \text { otherwise. }
\end{array}\right.
$$

Example: Given the following bba defined on $\Theta=\left\{\theta_{1}, \theta_{2}, \theta_{3}\right\}$ as:

$m^{\Theta}\left(\left\{\theta_{1}\right\}\right)=0.5, m^{\Theta}\left(\left\{\theta_{2}\right\}\right)=0.2$ and $m^{\Theta}(\{\Theta\})=0.3$.

Let $\Omega=\left\{\omega_{1}, \omega_{2}\right\}$.

The bba defined on $\Theta$ will be defined in a finer frame $\Theta \times \Omega$ using the vacuous extension as follows:

$$
\begin{aligned}
& m^{\Theta \uparrow \Theta \times \Omega}\left(\left\{\left(\theta_{1}, \omega_{1}\right),\left(\theta_{1}, \omega_{2}\right)\right\}\right)=0.5 \\
& m^{\Theta \uparrow \Theta \times \Omega}\left(\left\{\left(\theta_{2}, \omega_{1}\right),\left(\theta_{2}, \omega_{2}\right)\right\}\right)=0.2 \\
& m^{\Theta \uparrow \Theta \times \Omega}(\Theta \times \Omega)=0.3
\end{aligned}
$$

\subsubsection{Marginalization}

Given a mass distribution defined on the product space $\Theta \times \Omega$, marginalization corresponds to mapping over a subset of the product space by dropping the extra coordinates. The new belief defined on $\Theta$ is obtained by ${ }^{21}$ :

$$
m^{\Theta \times \Omega \downarrow \Theta}(A)=\sum_{\left.\left\{B \subseteq \Theta \times \Omega \mid B^{\downarrow \Theta}=A\right)\right\}} m^{\Theta \times \Omega}(B), \forall A \subseteq \Theta .
$$

$B^{\downarrow \Theta}$ denotes the projection of $B$ onto $\Theta$.

Example: Let us consider the bba defined on $\Theta \times \Omega$ :

$$
m^{\Theta \times \Omega}\left(\left\{\left(\theta_{1}, \omega_{1}\right),\left(\theta_{1}, \omega_{2}\right)\right\}\right)=0.5
$$


$m^{\Theta \times \Omega}\left(\left\{\left(\theta_{2}, \omega_{1}\right),\left(\theta_{2}, \omega_{2}\right)\right\}\right)=0.2$

$m^{\Theta \times \Omega}\left(\left\{\left(\theta_{1}, \omega_{1}\right)\right\}\right)=0.3$

Marginalizing $m^{\Theta \times \Omega}$ on the coarser frame $\Theta m^{\Theta \times \Omega \downarrow \Theta}$ will lead to the following distribution:

$$
\begin{aligned}
& m^{\Theta \times \Omega \downarrow \Theta}\left(\left\{\left(\theta_{1}\right\}\right)=0.5+0.3=0.8\right. \\
& m^{\Theta \times \Omega \downarrow \Theta}\left(\left\{\left(\theta_{2}\right\}\right)=0.2\right.
\end{aligned}
$$

\subsubsection{Ballooning extension.}

This operation is useful if an agent after conditioning realizes that the evidence he has considered as true was not and accordingly he would reconstruct the initial distribution. It can also be useful if beliefs are defined on a limited set and other alternatives were discovered afterwards. The agent should redistribute his beliefs to take them into account.

Let $m^{\Theta}[\omega]$ represents your beliefs on $\Theta$ conditionnally on $\omega$ a subset of $\Omega$. To get rid of conditioning, we have to compute its ballooning extension.

Conditional masses are transferred to $C$, the largest subset of $\Theta \times \Omega$ whose intersection with the vacuous extension of $\omega$ followed by a projection on $\Theta$ gives $A$ : $\left(A \cap \omega^{\uparrow \Theta \times \Omega}\right)^{\downarrow \Theta}=$ A.

Thus, $C=(A \times \omega \cup \Theta \times \bar{\omega})$ where $\bar{\omega}$ stands for the complement of $\omega$.

Accordingly, the ballooning extension is defined as:

$$
m^{\Theta}[\omega]^{\Uparrow \Theta \times \Omega}(A \times \omega \cup \Theta \times \bar{\omega})=m^{\Theta}[\omega](A), \forall A \subseteq \Theta .
$$

\section{Example:}

Let us consider $\Theta=\left\{\theta_{1}, \theta_{2}, \theta_{3}\right\}, \Omega=\left\{\omega_{1}, \omega_{2}\right\}$ and the conditional bba $m^{\Theta}\left[\omega_{1}\right]\left(\theta_{1}\right)=$ 0.6 . Its corresponding basic belief mass on $\Theta \times \Omega$ is obtained by taking into consideration $\left\{\left(\theta_{1}, \omega_{1}\right)\right\}$ and all the instances of $\Theta$ for the complement of $\omega_{1}$.

Hence, $m^{\Theta}\left[\omega_{1}\right]^{\Uparrow \Theta \times \Omega}\left(\left\{\left(\theta_{1}, \omega_{1}\right),\left(\theta_{1}, \omega_{2}\right),\left(\theta_{2}, \omega_{2}\right),\left(\theta_{3}, \omega_{2}\right)\right\}\right)=m^{\Theta}\left[\omega_{1}\right]\left(\theta_{1}\right)$.

\section{Belief function methods for generating belief function from preference relations}

The problem of eliciting qualitatively expert opinions and generating basic belief assignments has been addressed by many researchers 20232425 . In this subsection, we introduce some existing methods and we provide an overview of our proposed approach.

\subsection{Wong and Lingras' method}

Wong and Lingras ${ }^{25}$ proposed a method for generating belief functions from preference assessments. So, given a pair of propositions, experts may express which of the propositions is more likely to be true. Thus, they defined two binary relations preference $\succ$ and indifference $\sim$ defined on $2^{\Theta}$ such as:

$$
\begin{aligned}
& a \succ b \text { is equivalent to } \operatorname{bel}(a)>\operatorname{bel}(b) \\
& a \sim b \text { is equivalent to } \operatorname{bel}(a)=\operatorname{bel}(b)
\end{aligned}
$$


where $a, b \in 2^{\Theta}$.

This approach is based on two steps. The first one consists in considering that all the propositions that appear in the preference relations are potential focal elements. However, some propositions are eliminated according to the following condition: if $a \sim b$ for some $a \subset b$, then $a$ is not a focal element.

After that, the basic belief assignment is generated using the two presented Equations (12) and (13). This formulation has multiple belief functions that are consistent with the input qualitative information, and so their procedure only generates one of them.

It should be noted that Wong and Lingras' approach does not address the issue of inconsistency in the pair-wise comparisons. For example, the expert could specify the apparently inconsistent preference relationships: $\operatorname{bel}(a)>\operatorname{bel}(b), \operatorname{bel}(b)>\operatorname{bel}(c)$, and $\operatorname{bel}(c)>\operatorname{bel}(a)$

\subsection{Ben Yaghlane et al.'s method}

Ben Yaghlane et al. proposed a method for generating optimized belief functions from qualitative preferences. ${ }^{23}$ The objective of this method is then to convert preference relations into constraints of an optimization problem whose resolution, according to some uncertainty measures (UM), allows the generation of the least informative or the most uncertain belief functions defined as follows:

$$
\begin{gathered}
a \succ b \Rightarrow \operatorname{bel}(a)-\operatorname{bel}(b) \geq \varepsilon \\
a \sim b \Rightarrow|\operatorname{bel}(a)-\operatorname{bel}(b)| \leq \varepsilon
\end{gathered}
$$

where $\varepsilon$ is considered to be the smallest gap that the expert may discern between the degrees of belief in two propositions $a$ and $b$. Note that $\varepsilon$ is a constant specified by the expert before beginning the optimization process.

Ben Yaghlane et al. proposed a method that requires that propositions be represented in terms of focal elements, and they assume that $\Theta$ (where $\Theta$ is the frame of discernment) should always be considered as a potential focal element. Then, a mono-objective technique was used to solve such constrained optimization problem:

$$
\begin{gathered}
\operatorname{Max}_{m} U M(m) \\
\text { s.t. } \\
\operatorname{bel}(a)-\operatorname{bel}(b) \geq \varepsilon \\
\operatorname{bel}(a)-\operatorname{bel}(b) \leq \varepsilon \\
\operatorname{bel}(a)-\operatorname{bel}(b) \geq-\varepsilon \\
\sum_{a \in \mathcal{F}(m)} m(a)=1, m(a) \geq 0, \forall a \subseteq \Theta ; m(\emptyset)=0
\end{gathered}
$$

where the first, second and third constraints are derived from Equations (14) and (15), representing the quantitative constraints corresponding to the preference relations.

Furthermore, the proposed method addresses the problem of inconsistency. In fact, if the preference relations are consistent, then the optimization problem is feasible. Otherwise no solutions will be found. Thus, the expert may be guided to reformulate his preferences. 


\section{MCDM method based on belief preference relations}

This section is dedicated to the presentation of our new MCDM under uncertainty. Indeed, we introduce the basic stages needed to ensure the ranking of alternatives in an uncertain environment based on the belief function framework. In this context, we introduce a new method inspired of the AHP approach. Our model has the same features as standard AHP such as hierarchical levels and pair-wise comparisons. At first, we will briefly describe Saaty's approach. Then, we will present the computational steps of our proposed model.

\subsection{Aspects of analytic hierarchy process}

The objective of this section is to introduce the main ideas behind the AHP approach, in order to better introduce our approach which is inspired of it. In fact, our method will be based primarily on hierarchical level and pair-wise comparisons.

The AHP approach is a decision-making technique developed by Saaty. ${ }^{7} 8$ This method has the advantage of permitting a hierarchical structure of the criteria and alternatives, which provides users with a better focus on specific criteria and sub-criteria and alternatives when allocating the priorities. This step is important, because a different structure may lead to a different final ranking.

Once the hierarchy is built, the decision maker starts the prioritization procedure to determine the relative importance of the elements on each level of the hierarchy (criteria and alternatives). Elements of a problem on each level are paired (with respect to their upper level decision elements) and then compared. This method elicits preferences through pair-wise comparisons which are constructed from decision maker's answers. Indeed, the decision maker can use both objective information about the elements as well as subjective opinions about the elements relative meaning and importance. The responses to the pairwise comparison question use a nine-point scale ${ }^{7}$, which translates the preferences of a decision maker into crisp numbers.

After filling all the pair-wise comparison matrices, the objective is then to find the weight of each criterion, or the score of each alternative by calculating the eigenvalue vector. With these values, the AHP method permits to compute a consistency ratio to check if the matrix is consistent or not. When the matrix is considered inconsistent, the entries that are given by the decision maker have to be revised until a satisfactory consistency ratio is obtained.

\subsection{MCDM method using preferential assessments}

A MCDM problem is defined as a set of alternatives $\Theta=\left\{a_{1}, \ldots, a_{n}\right\}$, a set of criteria $\Omega=\left\{c_{1}, \ldots, c_{m}\right\}$, a preferential assessment representing the performance of each alternative with respect to each criterion leading to the determination of a decision matrix for the alternatives, and a weighting vector representing the relative importance of the evaluation criteria with respect to the overall objective of the problem. With regard to this problem, a new MCDM method has been developed. It is inspired of standard AHP approach and the belief function framework. 


\subsection{Identification of the candidate alternatives and criteria}

The ranking procedure starts at the determination of the criteria importance and alternative performance. As in ${ }^{5}$, let $\Omega=\left\{c_{1}, \ldots, c_{m}\right\}$ be a set of criteria, we denote the set of all subsets of $\Omega$ by $2^{\Omega}$, and let $C_{k}$ be the short notation of a subset of $\Omega$. Besides, in many complex problems decision makers are able to compare only subsets of criteria and cannot evaluate separate ones. To solve this problem, that means to reduce the number of criteria which decreases the number of comparisons, our method suggests to allow the expert to express his opinions on groups of criteria instead of single one. So, he chooses these subsets by assuming that criteria having the same degree of preference are grouped together. For instance, if an expert identifies a group of criteria, then we could suppose that all of them have the same importance. Thus, these groups of criteria can be defined as:

$$
C_{k} \succ C_{j}, \forall k, j \mid C_{k}, C_{j} \in 2^{\Omega}, C_{k} \cap C_{j}=\emptyset \text { and } \bigcup_{j} C_{j}=\Omega \quad \text { (with } C_{j} \text { exclusive). }
$$

Since we are not performing pair-wise comparisons of criteria but relating to groups of criteria, these sets should not consider a criterion in common, because if one criterion is included in two groups, then each group will give a different level of favorability.

For example, we consider a problem that involves four criteria: $\Omega=\left\{\operatorname{Comfort}\left(c_{1}\right)\right.$, Style $\left(c_{2}\right)$, Fuel $\left(c_{3}\right)$, Quietness $\left.\left(c_{4}\right)\right\}$. The expert can identify the following subsets $\left\{c_{1}\right\}$, $\left\{c_{4}\right\}$, and $\left\{c_{2}, c_{3}\right\}$. He assumed that the two criteria $c_{2}$ and $c_{3}$ have the same degree of importance. Therefore, they are grouped together.

Like the criterion level, we assume that there is a set of alternatives $\Theta=\left\{a_{1}, \ldots, a_{n}\right\}$ consisting of $n$ elements. Denote the set of all subsets of $\Theta$ by $2^{\Theta}$, and let $A_{k}$ be a subset of $A$. At this level, our method suggests to not necessarily consider all of them but just to choose groups of those alternatives. One of the possible solutions of this task is to use the DS/AHP method. ${ }^{13} 14$ Besides, we apply the same hypotheses assumed in DS/AHP to identify the subsets of alternatives. The decision maker compares not only a single one but also sets of alternatives between each other.

By comparing subsets of criteria and alternatives, we provide a major benefit to the expert. Our proposed approach reduces the number of comparisons, because instead of using single elements, we have used subsets. In fact, in some practical problems, it is easier for an expert to express his opinions and comparisons between subsets of criteria and not compulsory on singleton criterion. For example, for the fuel, we may get two subgroups: the first one containing both natural gas and gasoline criteria and the second one only singleton criterion which is diesel.

\subsection{Pair-wise comparisons and preference elicitation}

Once the sets of criteria and alternatives are defined, the expert tries to specify his preferences in order to obtain the criterion weights and the alternative performances in terms of each criterion. At this step, we propose to use the pair-wise comparison process.

Under this approach, a new elicitation procedure is introduced, since standard pairwise comparison does not handle the problem of uncertainty. Therefore, in the proposed 
methodology, the expert is allowed to use preference relations only. Thus to express his assessments, the decision maker has to express his opinions qualitatively, based on knowledge and experience that he provides in response to a given question rather than direct quantitative information. He only selects the related linguistic variable using preference modeling.

For instance, to determine the criteria weights, the preferences relation matrix is obtained (Table 1).

Table 1. Preferences relation matrix.

\begin{tabular}{|c|c|c|c|c|}
\hline & $c_{1}$ & $c_{2}$ & $\ldots$ & $c_{m}$ \\
\hline$c_{1}$ & - & $P_{12}$ & $\ldots$ & $P_{1 m}$ \\
$c_{2}$ & - & - & $\ldots$ & $P_{2 m}$ \\
$\ldots$ & - & - & - & $\ldots$ \\
$c_{m}$ & - & - & - & - \\
\hline
\end{tabular}

In this table, $P_{i j}$ is a preference relations. It may be:

(1) a preference relation $\succ$ iff $\left(c_{i} \succ c_{j}\right) \wedge \neg\left(c_{j} \succ c_{i}\right)$

(2) an indifference relation $\sim$ iff $\left(c_{i} \succ c_{j}\right) \wedge\left(c_{j} \succ c_{i}\right)$

(3) an unknown relation.

To complete the pair-wise matrix, the expert is not obliged to quantify his assessments and to fill all the pair-wise comparisons matrix. He is able to express his preferences freely.

To model the pair-wise comparison matrix, some priorities must be respected. We consider $X$, the pair-wise comparison matrix, is a $k \times k$ matrix in which $k$ is the number of groups of elements (alternatives or criteria) being compared. Entries of $X$ denote the preference relation between each pair of elements. The expert has to complete only the matrix without quantifying the diagonal and the reciprocal matrix.

Once the pair-wise comparison matrix is complete for both the criterion and the alternative levels, our objective is then to obtain the priority of each subset of element (alternatives or criteria). In fact, within our model, we propose to transform these preference relations into numerical values using the belief function theory. Besides, we try to closely imitate the expert reasoning without adding any additional information. Therefore, we suggest to apply Ben Yaghlane et al. model ${ }^{23}$ to convert the preference relations into constraints of an optimization problem whose resolution, according to some uncertainty measures (UM), allows the generation of the least informative or the most uncertain belief functions. It can then be determined by the resolution of an optimization problem as defined is the previous section (Equation 16).

Using the obtained pair-wise matrix, each preference relation is transformed into constraints of an optimization problem. For instance, if we use the preferences relation matrix relative to the criterion level we get: 


$$
\begin{gathered}
\operatorname{Max}_{m} H(m)=m\left(\left\{c_{1}\right\}\right) * \log _{2}\left(\left|c_{1}\right| / m\left(\left\{c_{1}\right\}\right)\right)+m\left(\left\{c_{2}\right\}\right) \log _{2}\left(\left|c_{2}\right| / m\left(\left\{c_{2}\right\}\right)\right) \\
+\ldots+m\left(\left\{c_{m}\right\}\right) * \log _{2}\left(\left|c_{m}\right| / m\left(\left\{c_{m}\right\}\right)\right)+m(\Omega) * \log _{2}(|\Omega| / m(\Omega)) ; \\
\text { s.t. } \\
\operatorname{bel}\left(\left\{c_{1}\right\}\right)-\operatorname{bel}\left(\left\{c_{2}\right\}\right) \geq \varepsilon \quad\left(c_{1} \text { is prefered to } c_{2}\right) \\
\operatorname{bel}\left(\left\{c_{1}\right\}\right)-\operatorname{bel}\left(\left\{c_{2}\right\}\right) \geq-\varepsilon \quad\left(c_{1} \text { is indifferent to } c_{2}\right) \\
\operatorname{bel}\left(\left\{c_{1}\right\}\right)-\operatorname{bel}\left(\left\{c_{2}\right\}\right) \leq \varepsilon \quad\left(c_{1} \text { is indifferent to } c_{2}\right) \\
\ldots \\
\sum_{c_{i} \in \mathcal{F}(m)} m\left(c_{i}\right)=1, m(A) \geq 0, \forall A \subseteq \Omega ; m(\emptyset)=0 .
\end{gathered}
$$

We assume that $\varepsilon=0.01$ and the uncertainty measures is $H$ since it has a unique maximum as defined in Equation 6. In this paper the measure $H$ is used since it takes into account the non-specificity and quantifies the conflict presented in the body of evidence (measure of total uncertainty). Among these measures, we decided to use $\mathrm{H}$.

As a result, we assume that each subset is described by a basic belief assignment defined on the possible responses $(m)$. Thus, the criterion bba is denoted by $m^{\Omega}$ and the alternative bba by $m^{\Theta}$.

Furthermore, the proposed method addresses the problem of inconsistency. In fact, if the preference relations are consistent, then the optimization problem is feasible. Otherwise no solution will be found.

\subsection{Updating the alternatives priorities}

To update the alternatives priorities with respect to the criterion weight, we have to define a rule for combining them. On the one hand, we have bba concerning singleton and groups of criteria instead of single one. On the other hand, the sets of alternatives are compared pair-wise regarding a specific criterion. ${ }^{28}$

Accordingly, and to more imitate the expert reasoning, we propose to represent the relationship between criteria alternative level. Unlike the criterion level, the expert tries to express his preferences over the sets of alternatives regarding each criterion and not regardless of the criteria. Besides, we indicate that to define the influences of the criteria on the evaluation of alternatives, we might use a conditional belief.

Given a pair-wise comparison matrix which compares the sets of alternatives according to a specific criterion, a conditional bba can be represented by: ${ }^{28}$

$$
m^{\Theta}\left[c_{j}\right]\left(A_{k}\right)=w_{k}, \quad \forall A_{k} \subseteq \Theta \text { and } c_{j} \in \Omega
$$

where $A_{k}$ represents a subset of $2^{\Theta}, w_{k}$ is the bbm of the $k^{t h}$ sets of alternatives regarding the criterion $c_{j} \cdot m^{\Theta}\left[c_{j}\right]\left(A_{k}\right)$ means that we know the belief about $A_{k}$ regarding $c_{j}$.

As indicated above, our objective through this step is to combine the obtained conditional belief with the importance of their respective criteria to measure their contribution. In this context, our major problem here is that we have priorities concerning criteria and groups of criteria that are defined on the frame of discernment $\Omega$, whereas the sets of decision alternatives are generally defined on another frame $\Theta$. In order to solve this problem, 
we propose to standardize our frame of discernment. First, at the criterion level, our objective is then to redefine the bba that represents criteria weights. Therefore, we may use the vacuous extension concept (Equation 9). ${ }^{21}$ Indeed, we propose to extend this bba from $\Omega$ to $\Theta \times \Omega$ :

$$
m^{\Omega \uparrow \Theta \times \Omega}(B)=m^{\Omega}\left(C_{i}\right) \quad B=\Theta \times C_{i}, C_{i} \subseteq \Omega .
$$

Second, at the alternative level, the idea was to use the deconditionalization process in order to transform the conditional belief into a new belief function. In this case, the ballooning extension concept (Equation 11) is applied: ${ }^{21}$

$$
m^{\Theta}\left[c_{j}\right]^{\Uparrow \Theta \times \Omega}\left(A_{k} \times c_{j} \cup \Theta \times \overline{c_{j}}\right)=m^{\Theta}\left[c_{j}\right]\left(A_{k}\right), \forall A_{k} \subseteq \Theta .
$$

Once the frame of discernment $\Theta \times \Omega$ is formalized, our approach proposes to combine the alternative priorities. In fact, we assume that each pair-wise comparison matrix is considered as a distinct source of evidence, which provides opinions towards the preferences of particular decision alternatives. Then, based on the belief function framework, we can apply the conjunctive rule of combination. The obtained bba represents the belief in groups of alternatives based on the combined evidence from the decisions matrices.

Finally, we might combine the obtained bba with the importance of their respective criteria to measure their contribution. That is, we will apply the conjunctive rule of combination and we get:

$$
m^{\Theta \times \Omega}=\left[\bigcirc_{j=1, \ldots, m} m^{\Theta}\left[c_{j}\right]^{\Uparrow \Theta \times \Omega}\right] @ m^{\Omega \uparrow \Theta \times \Omega} .
$$

So, we obtain $m^{\Theta \times \Omega}$ reflecting the importance of alternatives to the given criteria.

\subsection{Decision making}

To this end and after combining the resulting ballooning extension, a decision under uncertainty must be defined. In the sequel, the pignistic probabilities are used. However, our obtained beliefs are defined on the product space $\Theta \times \Omega$. To solve this problem, we propose to marginalize this bba on $\Theta$ (frame of alternatives) by transferring each mass $m^{\Theta \times \Omega}$ to its projection on $\Theta$ (Equation 8):

$$
m^{\Theta \times \Omega \downarrow \Theta}\left(A_{j}\right)=\sum_{\left.\left\{B \subseteq \Theta \times \Omega \mid \operatorname{Proj}(B \downarrow \Theta)=A_{j}\right)\right\}} m^{\Theta \times \Omega}(B), \forall A_{j} \subseteq \Theta .
$$

Finally, we can compute the pignistic probabilities to choose the best alternatives:

$$
\operatorname{BetP}\left(a_{j}\right)=\sum_{A_{i} \subseteq \Theta} \frac{\left|a_{j} \cap A_{i}\right|}{\left|A_{i}\right|} \frac{m^{\Theta \times \Omega \downarrow \Theta}\left(A_{i}\right)}{\left(1-m^{\Theta \times \Omega \downarrow \Theta}(\emptyset)\right)}, \forall a_{j} \in \Theta .
$$

\subsection{Example}

In this section, we present an illustrative example showing that the proposed approach is a convenient tool for ranking alternatives in an uncertain environment based on belief assessments and qualitative evaluations. 
We present an empirical study of purchasing a car. ${ }^{28}$ In fact, selecting the best car from available alternatives is a classical and complex decision-making problem in which the overall performance of the alternatives needs to be evaluated with respect to each identified criteria. The reason why, subjective and qualitative assessments are often involved with regard to the criteria weight and alternatives score, resulting the use of the belief function framework to elicitate the decision maker preferences.

The first stage is the identification of the candidate criteria and alternatives. Our problem involves four criteria: $\Omega=\left\{\right.$ Comfort $\left(c_{1}\right)$, Style $\left(c_{2}\right)$, Fuel $\left(c_{3}\right)$, Quietness $\left.\left(c_{4}\right)\right\}$, and three selected alternatives: $\Theta=\{$ Peugeot $(p)$, Renault $(r)$, Ford $(f)\}$.

Along with our MCDM Method using preferential assessments, a judgment matrix based on the pair-wise comparison process using preference modeling defined in Table 2 was obtained as follows. So, given the necessary details of the criteria, the decision maker was asked to indicate his level of preference between them. Importantly, he was made aware that criteria that belongs to the same subset have the same importance.

Table 2. Preference relation matrix for criterion level

\begin{tabular}{|c|c|c|c|}
\hline Criteria & $\left\{c_{1}\right\}$ & $\left\{c_{4}\right\}$ & $\left\{c_{2}, c_{3}\right\}$ \\
\hline$\left\{c_{1}\right\}$ & - & $\succ$ & $\succ$ \\
$\left\{c_{4}\right\}$ & - & - & $\succ$ \\
$\left\{c_{2}, c_{3}\right\}$ & - & - & - \\
\hline
\end{tabular}

From Table 2, we conclude that the decision maker has identified three subsets of criteria $\left\{c_{1}\right\},\left\{c_{4}\right\}$, and $\left\{c_{2}, c_{3}\right\}$. For instance, expert may say that $\left\{c_{1}\right\}$ is evaluated to be more important than $\left\{c_{4}\right\}$ and $\left\{c_{1}\right\}$ is evaluated to be more preferred than $\left\{c_{2}, c_{3}\right\}$.

Now, for deriving the weights of criteria, we apply our presented model. Therefore, we must transform these qualitative assessments into an optimization problem (Equation 16) and we get the following constraints:

(1) $c_{1} \succ c_{4} \Leftrightarrow \operatorname{bel}\left(\left\{c_{1}\right\}\right)-\operatorname{bel}\left(\left\{c_{4}\right\}\right) \geq \varepsilon$

(2) $c_{1} \succ\left(c_{2}, c_{3}\right) \Leftrightarrow \operatorname{bel}\left(\left\{c_{1}\right\}\right)-\operatorname{bel}\left(\left\{c_{2}, c_{3}\right\}\right) \geq \varepsilon$

(3) $c_{4} \succ\left(c_{2}, c_{3}\right) \Leftrightarrow \operatorname{bel}\left(\left\{c_{4}\right\}\right)-\operatorname{bel}\left(\left\{c_{2}, c_{3}\right\}\right) \geq \varepsilon$

Then, the obtained constraints are transformed into optimization problem in order to obtain the importance of criteria. We assume that $\varepsilon=0.01$ and the uncertainty measure is $H$ as defined in Equation 6. 


$$
\begin{gathered}
\operatorname{Max}_{m} H(m)=m\left(\left\{c_{1}\right\}\right) * \log _{2}\left(1 / m\left(\left\{c_{1}\right\}\right)\right)+m\left(\left\{c_{4}\right\}\right) \log _{2}\left(1 / m\left(\left\{c_{4}\right\}\right)\right) \\
+m\left(\left\{c_{2}, c_{3}\right\}\right) * \log _{2}\left(2 / m\left(\left\{c_{2}, c_{3}\right\}\right)\right)+m(\Omega) * \log _{2}(4 / m(\Omega)) ; \\
\text { s.t. } \\
\operatorname{bel}\left(\left\{c_{1}\right\}\right)-\operatorname{bel}\left(\left\{c_{4}\right\}\right) \geq \varepsilon \\
\operatorname{bel}\left(\left\{c_{1}\right\}\right)-\operatorname{bel}\left(\left\{c_{2}, c_{3}\right\}\right) \geq \varepsilon \\
\operatorname{bel}\left(\left\{c_{4}\right\}\right)-\operatorname{bel}\left(\left\{c_{2}, c_{3}\right\}\right) \geq \varepsilon \\
\sum_{c_{i} \in \mathcal{F}(m)} m\left(c_{i}\right)=1, m(A) \geq 0, \forall A \subseteq \Omega ; m(\emptyset)=0 .
\end{gathered}
$$

Finally, Table 3 represents the weighting vector (the result is obtained using Matlab ${ }^{\circledR}$ toolbox).

Table 3. The weights assigned to the subset of criteria

\begin{tabular}{|c|c|c|c|c|}
\hline Criteria & $\left\{c_{1}\right\}$ & $\left\{c_{4}\right\}$ & $\left\{c_{2}, c_{3}\right\}$ & $\Omega$ \\
\hline$m^{\Omega}$ & 0.228 & 0.218 & 0.208 & 0.346 \\
\hline
\end{tabular}

In Table 3, a new subset of criteria is appeared, this group $\Omega=\left\{c_{1}, c_{2}, c_{3}, c_{4}\right\}$ expresses then the part of ignorance.

Similarly to the criterion level, the judgments between decision alternatives over different criteria are dealt within an identical manner. For example, to evaluate the alternatives according to the criterion $c_{1}$, the expert is asked to evaluate the following subsets of alternatives: $\{p\}$ and $\{p, r, f\}$ (see Table 4).

Table 4. Preferences relation matrix for alternative regarding $c_{1}$

\begin{tabular}{|c|c|c|c|}
\hline Criteria & $\{p\}$ & $\{p, r, f\}$ & bba \\
\hline$\{p\}$ & - & $\succ$ & 0.505 \\
$\{p, r, f\}$ & - & - & 0.495 \\
\hline
\end{tabular}

As similar process is repeated and a comparison judgment matrix in regard to each identified criterion was obtained (Table 5).

Table 5. Priorities values

\begin{tabular}{|c|c||c|c||c|c||c|c|}
\hline$c_{1}$ & $m^{\Theta}\left[c_{1}\right]()$. & $c_{2}$ & $m^{\Theta}\left[c_{2}\right]()$. & $c_{3}$ & $m^{\Theta}\left[c_{3}\right]()$. & $c_{4}$ & $m^{\Theta}\left[c_{4}\right]()$. \\
\hline$\{p\}$ & 0.505 & $\{p\}$ & 0.319 & $\{r\}$ & 0.505 & $\{f\}$ & 0.505 \\
$\{p, r, f\}$ & 0.495 & $\{r, f\}$ & 0.535 & $\{p, r, f\}$ & 0.495 & $\{p, r, f\}$ & 0.495 \\
& & $\{p, r, f\}$ & 0.146 & & & & \\
\hline
\end{tabular}

After computing the belief functions for each set of alternatives with respect to each criterion, we must combine the weight of criteria and the alternatives priorities. Firstly, 
our presented approach proposes to standardize the criterion and the alternative frames of discernment. For the criterion level, we suggest to apply the extension procedure. Hence, Equation 20 is used and the resulting bba's are summarized in Table 6.

Table 6. Vacuous extension of bba

\begin{tabular}{|c|c|c|}
\hline$m^{\Omega}()$. & $m^{\Omega \uparrow \Theta \times \Omega}$ & bbm \\
\hline$m^{\Omega}\left(\left\{c_{1}\right\}\right)$ & $\left\{\left(p, c_{1}\right),\left(r, c_{1}\right),\left(f, c_{1}\right)\right\}$ & 0.228 \\
\hline$m^{\Omega}\left(\left\{c_{4}\right\}\right)$ & $\left\{\left(p, c_{4}\right),\left(r, c_{4}\right),\left(f, c_{4}\right)\right\}$ & 0.218 \\
\hline$m^{\Omega}\left(\left\{c_{2}, c_{3}\right\}\right)$ & $\left\{\left(p, c_{2}\right),\left(r, c_{2}\right),\left(f, c_{2}\right),\left(p, c_{3}\right),\left(r, c_{3}\right),\left(f, c_{3}\right)\right\}$ & 0.208 \\
\hline$m^{\Omega}(\Omega)$ & $\left\{\left(p, c_{1}\right),\left(r, c_{1}\right),\left(f, c_{1}\right),\left(p, c_{2}\right),\left(r, c_{2}\right),\left(f, c_{2}\right),\left(p, c_{3}\right),\left(r, c_{3}\right),\left(f, c_{3}\right)\right\}$ & 0.346 \\
\hline
\end{tabular}

After that, the next step concerns the alternative level. In fact, our method suggests to transform the conditional belief into joint distribution. For instance, if we present the priority matrix that evaluates the candidate subsets of alternatives regarding the criterion $c_{1}$ and by applying the ballooning extension using Equation 21.

Let us consider for example, $m^{\Theta}\left[c_{1}\right](\{p\})$ its corresponding basic belief mass on $\Theta \times \Omega$ is obtained by $\left\{\left(p, c_{1}\right)\right\}$ and all the instances of $\Theta\left\{\left(p, c_{1}\right),\left(p, c_{2}\right),\left(p, c_{3}\right),\left(p, c_{4}\right)\right\}$ for the complement of $c_{1}\left\{\left(r, c_{2}\right),\left(r, c_{3}\right),\left(r, c_{4}\right),\left(f, c_{2}\right),\left(f, c_{3}\right),\left(f, c_{4}\right)\right\}$. The following Table 7 is calculated.

Table 7. Ballooning extension of conditional bba

\begin{tabular}{|c|c|c|}
\hline$m^{\Theta}\left[c_{1}\right]()$. & $m^{\Theta}\left[c_{j}\right]^{\Uparrow \Theta \times \Omega}$ & bbm \\
\hline$m^{\Theta}\left[c_{1}\right](\{p\})$ & $\begin{array}{l}\left\{\left(p, c_{1}\right),\left(p, c_{2}\right),\left(p, c_{3}\right),\left(p, c_{4}\right),\left(r, c_{2}\right),\right. \\
\left.\left(r, c_{3}\right),\left(r, c_{4}\right),\left(f, c_{2}\right),\left(f, c_{3}\right),\left(f, c_{4}\right)\right\}\end{array}$ & 0.505 \\
\hline$m^{\Theta}\left[c_{1}\right](\{p, r, f\})$ & $\begin{array}{c}\left\{\left(p, c_{1}\right),\left(p, c_{2}\right),\left(p, c_{3}\right),\left(p, c_{4}\right),\right. \\
\left.\left(r, c_{1}\right),\left(r, c_{2}\right),\left(r, c_{3}\right),\left(r, c_{4}\right),\left(f, c_{1}\right),\left(f, c_{2}\right),\left(f, c_{3}\right),\left(f, c_{4}\right)\right\}\end{array}$ & 0.495 \\
\hline
\end{tabular}

Then, similar process is repeated for the rest of alternatives regarding each criterion.

Finally, the obtained bba's $m^{\Omega \uparrow \Theta \times \Omega}$ and $m^{\Theta}\left[c_{j}\right]^{\Uparrow \Theta \times \Omega}$ can be directly combined using the conjunctive rule of combination to get the Table 8 .

To choose the best alternatives, we must define our beliefs over the frame of alternatives. As a result, the obtained bba is marginalized on $\Theta$ using the Equation 23 (see Table 9).

We can now calculate the overall performance for each alternative and determine its corresponding ranking by computing the pignistic probabilities (Table 10).

As a consequence, the alternative "Peugeot" is the recommended car since it has the highest values. This research proposes that the difference between standard AHP method and the proposed approach are raised by a major factor. Our method can provide the decision maker to express his preferences with some uncertainty. In fact, our model allows the expert to use preferential assessments rather than deterministic value options in order 
Table 8. The obtained bba: $m^{\Theta \times \Omega}$

\begin{tabular}{|c|c|}
\hline$m^{\Theta \times \Omega}$ & bbm \\
\hline$\left\{\left(p, c_{1}\right),\left(f, c_{1}\right),\left(r, c_{1}\right)\right\}$ & 0.377 \\
\hline$\left\{\left(p, c_{1}\right)\right\}$ & 0.225 \\
\hline$\left\{\left(p, c_{4}\right),\left(f, c_{4}\right),\left(r, c_{4}\right)\right\}$ & 0.18 \\
\hline$\left\{\left(f, c_{1}\right)\right\}$ & 0.005 \\
\hline$\left\{\left(p, c_{2}\right),\left(f, c_{2}\right),\left(r, c_{2}\right),\left(p, c_{3}\right),\left(f, c_{3}\right),\left(r, c_{3}\right)\right\}$ & 0.003 \\
\hline$\left\{\left(r, c_{2}\right),\left(r, c_{3}\right),\left(f, c_{2}\right),\left(p, c_{2}\right)\right\}$ & 0.1 \\
\hline$\emptyset$ & 0.11 \\
\hline
\end{tabular}

Table 9. The obtained bba: $m^{\Theta \times \Omega \downarrow \Theta}$

\begin{tabular}{|c|c|}
\hline$m^{\Theta \times \Omega \downarrow \Theta}$ & bbm \\
\hline$m^{\Theta \times \Omega \downarrow \Theta}(\{p, r, f\})$ & 0.66 \\
\hline$m^{\Theta \times \Omega \downarrow \Theta}(\{p\})$ & 0.225 \\
\hline$m^{\Theta \times \Omega \downarrow \Theta}(\{f\})$ & 0.005 \\
\hline$m^{\Theta \times \Omega \downarrow \Theta}(\emptyset)$ & 0.11 \\
\hline
\end{tabular}

Table 10. The Final ranking of alternative

\begin{tabular}{|c|c|c|c|}
\hline Alternatives & $\{p\}$ & $\{r\}$ & $\{f\}$ \\
\hline BetP & 0.5 & 0.253 & 0.247 \\
\hline Ranking & 1 & 2 & 3 \\
\hline
\end{tabular}

to more imitate his reasoning. These evaluations are expressed using belief function framework.

For the sake of comparison, we have used belief AHP method to treat the same problem. 28 The same results were obtained as shown in Table 11. It is clear that "Peugeot" is the best choice. Belief AHP method and the approach developed have obtained the same best alternative. This would give the expert reasonable assurance in decision making.

Table 11. The Final ranking of alternative using belief AHP

\begin{tabular}{|c|c|c|c|}
\hline Alternatives & $\{p\}$ & $\{r\}$ & $\{f\}$ \\
\hline BetP & 0.567 & 0.213 & 0.220 \\
\hline Ranking & 1 & 3 & 2 \\
\hline
\end{tabular}

However in comparison with the Belief AHP method, the approach developed clearly has its advantages. In fact, our MCDM method is able to model uncertainty and imprecision associated with the pair-wise comparison process and it is able to quantify the criterion 
weights and the alternative scores without involving any numerical values to provide them.

\section{Conclusion}

In this paper, we have proposed a MCDM method in an environment characterized by the imperfection of the information. The belief function framework has been used for modeling the uncertainty related to the expert assessment and also to represent the decision maker preferences qualitatively without using any numerical values. The expert is then allowed to freely express his assessments using belief preferences relations only. Therefore, the originality of our model is its ability to express the expert opinions freely and based on incomplete ones. Also it can rank alternatives based on qualitative preferences only. On one hand, our proposed method models uncertainty by expressing the expert assessments using bba. On the other hand, it represents experts impression by comparing groups of criteria and alternatives instead of single ones.

\section{References}

1. P. Joaquin, "Some Comments on the Analytic Hierarchy Process", Journal of the Operational Research Society 41(6) (1990) 1073-1076.

2. R. D. Holder, "Some Comments on Saaty's AHP”, Management Science 41 (1995) 1091-1095.

3. G. Shafer, "A Mathematical Theory of Evidence", Princeton University Press, 1976.

4. Ph. Smets, "The Application of the Transferable Belief Model to Diagnostic Problems", International Journal of Intelligent Systems 13 (1998) 127-158.

5. A. Ennaceur, Z. Elouedi and E. Lefevre, "Handling Partial Preferences in the Belief AHP Method: Application to Life Cycle Assessment", Proceedings of the International Conference of the Italian Association for Artificial Intelligence (IA*AI), LNAI 6934, Springer-Verlag (2011) pp. 396-401.

6. M. Zeleny, "Multiple Criteria Decision Making", McGraw-Hill Book Company, 1982.

7. T. Saaty, "A scaling method for priorities in hierarchical structures", Journal of Mathematical Psychology 15 (1977) 234-281.

8. T. Saaty, "The Analytic Hierarchy Process", McGraw-Hill, New-York, 1980.

9. P. J. M. Van Laarhoven and W. Pedrycz, "A fuzzy extension of Saaty's priority theory”, Fuzzy Sets and Systems 11 (1983) 199-227.

10. F. A. Lootsma, "Fuzzy Logic for planning and Decision-Making", Kluwer Academic Publishers, 1997.

11. I. Basak, "Probabilistic judgments specified partially in the Analytic Hierarchy Process", European Journal of Operational Research 108 (1998) 153-164.

12. B. Schoner and W. C. Wedley, "Ambiguous criteria weights in AHP: consequences and solutions", Decision Sciences 20 (1989) 462-475.

13. M. Beynon, B. Curry, and P. Morgan, "The Dempster-Shafer theory of evidence: An alternative approach to multicriteria decision modelling", OMEGA 28(1) (2000) 37-50.

14. M. Beynon, "DS/AHP method: A mathematical analysis, including an understanding of uncertainty", European Journal of Operational Research 140 (2002) 148-164.

15. F. Smarandache and J. Dezert, "Advances and Applications of DSmT for Information Fusion (Collected works)", American Research Press, 2004.

16. Ph. Smets, "The combination of evidence in the Transferable Belief Model", IEEE Pattern analysis and Machine Intelligence 12 (1990) 447-458.

17. Ph. Smets and R. Kennes, "The Transferable Belief Model", Artificial Intelligence 66 (1994) 191-234. 
18. L. V. Utkin, "A new ranking procedure by incomplete pairwise comparisons using preference subsets", Intelligent Data Analysis 13(2) (2009) 229-241.

19. L. V. Utkin and N. Simanova, "Multi-criteria decision making by incomplete preferences", Journal of Uncertain Systems, 2 (2008) 255-266.

20. A. Ennaceur, Z. Elouedi and E. Lefevre, "Introducing Incomparability in Modeling Qualitative Belief Functions", Proceedings of the International Conference on Modeling Decisions for Artificial Intelligence (MDAI), Springer-Verlag, Catalonia, Spain (2012) pp. 382-393.

21. Ph. Smets, "Belief functions: the disjunctive rule of combination and the generalized bayesian theorem", International Journal of Approximate Reasoning 9 (1990) 1-35.

22. J. Dezert, J. M. Tacnet, M. Batton-Hubert and F. Smarandache, "Multi-Criteria Decision Making based on DSmT-AHP", Workshop on the Theory of Belief Functions (2010).

23. A. Ben Yaghlane, T. Denoeux and K. Mellouli, "Constructing belief functions from expert opinions", Proceedings of the 2nd International Conference on Information and Communication Technologies: from Theory to Applications (ICTTA06), Damascus, Syria (2006) pp. 75-89.

24. N. Bryson and A. Mobolurin, "A process for generating quantitative belief functions", European Journal of Operational Research 115 (1999) 624-633.

25. S.K.M. Wong and P. Lingras, "Representation of Qualitative User Preference by Quantitative Belief Functions", IEEE Transactions on Knowledge and Data Engineering 6 (1994) 72-78.

26. N. Pal, J. Bezdek and R. Hemasinha, "Uncertainty measures for evidential reasoning I: A review", International Journal of Approximate Reasoning 7 (1992) 165-183.

27. N. Pal, J. Bezdek and R. Hemasinha, "Uncertainty measures for evidential reasoning II: A review", International Journal of Approximate Reasoning 8 (1993) 1-16.

28. A. Ennaceur, Z. Elouedi and E. Lefevre, "Reasoning under uncertainty in the AHP method using the belief function theory", The Fourteenth International Conference on Information Processing and Management of Uncertainty in Knowledge-Based Systems (IPMU), Catane, Italie (2012) pp. 373-382.

29. I. U. Sari, H. Behret and C. Kahraman, "Risk Governance of Urban Rail Systems Using Fuzzy AHP: The Case of Istanbul", International Journal of Uncertainty, Fuzziness and KnowledgeBased Systems 20 (2012) 67-79.

30. L. V. Utkin and N. V. Simanova, "The DS/AHP Method under Partial Information about Criteria and Alternatives By Several Levels of Criteria", International Journal of Uncertainty, Fuzziness and Knowledge-Based Systems 11(2) (2012) 307-326.

31. M. T. Lamata, and S. Moral, "Measures of entropy in the theory of evidence", International Journal of General Systems 14 (1987) 297-305.

32. G. J. Klir, and A. Ramer, "Uncertainty in Dempster Shafer theory: A critical re-examination,", International Journal of General Systems 18 (1990) 155-166. 